\title{
Genital pyoderma gangrenosum revealing Behçet's disease: a case report
}

\author{
Hassina Chicha, Said Taharboucht", Nacera Tiboune, Nadia Touati and Ahcene Chibane
}

\begin{abstract}
Background: Pyoderma gangrenosum $(\mathrm{PG})$ is a rare and chronic neutrophilic dermatosis. It is clinically characterized by aseptic ulcerations preferentially located in the lower limbs. Its location in the genital area is unusual and could be a source of diagnostic difficulties. In half of the cases, PG is associated with an underlying disease. The association with Behçet's disease is exceptional.

Case presentation: We report an original observation of a patient who presented a vulvar PG which revealed a Behçet's disease.

Conclusion: The distinction between these two pathologies was difficult because of the similarity of the cutaneousmucous lesions on the one hand, and the absence of histological specificity of these two pathologies on the other hand.
\end{abstract}

Keywords: Pyoderma gangrenosum, Behcet's disease, Pathergy test, Neutrophilic dermatoses

\section{Background}

Pyoderma gangrenosum (PG) is a rare, chronic neutrophilic dermatosis of unknown origin, its clinical appearance is an ulcerative pustular lesions [1]. Approximately $50 \%$ of PG cases are associated with an underlying systemic illness, in particularly inflammatory bowel disease such as ulcerative colitis or Crohn's disease [1]. Its occurrence during Behçet's disease (BD) is unusual [1]. Indeed, PG is cited among the most exceptional dermatological manifestations of $\mathrm{BD}$ which, as a reminder, is currently considered to be vasculitis affecting vessels of variable diameter [2]. We report a particular observation of a vulvar and fistulizing pyoderma gangrenosum which revealed Behçet's disease.

\section{Case presentation}

A 42-year-old female patient hospitalized for a painful genital ulceration evolving for 4 months. She had received several courses of appropriate antibiotic therapy without any improvement. On dermatological examination she

\footnotetext{
*Correspondence: staharboucht@yahoo.fr

Internal Medicine Department, University Saad Dahleb BLIDA 1, CHU de Douera, Algiers, Algeria
}

had a purulent oozing and stinky ulceration measuring $10 \mathrm{~cm}$, with ragged undermined edge, located at the level of the vulva with an extension to the perianal region. The margins were raised and purplish (Fig. 1). A pseudo folliculitis lesion was also found on the face (Fig. 2). Examination of the oral cavity revealed ulcers on the palate and tongue (Fig. 3). The patient reported the notion of recurrent oral aphthosis since 2 years. The pathergy test was strongly positive (Fig. 4). Ophthalmologic examination showed a bilateral intermediate and posterior uveitis associated with a strong Tyndall effect.

The diagnosis of $\mathrm{BD}$ was retained based on international criteria for Behçet's disease (Table 1) [3]. Bacteriological samples and repeated cultures of the ulceration were sterile. The laboratory examination objectified anemia at $9.9 \mathrm{~g} / \mathrm{dl}$ microcytic, hypochromic with an inflammatory syndrome (increased erythrocyte sedimentation and C-reactive protein (CRP at $93 \mathrm{mg} / \mathrm{l}$ ). The search for antinuclear and anti-DNA antibodies was negative, the Hepatitis B, C, HIV, and Syphilis viral serologies were negative. The chest X-ray and abdominal ultrasound were normal. Pelvic MRI found an anovaginal fistula with significant inflammatory changes in the anal margin and on the sphincter. The punch biopsy 


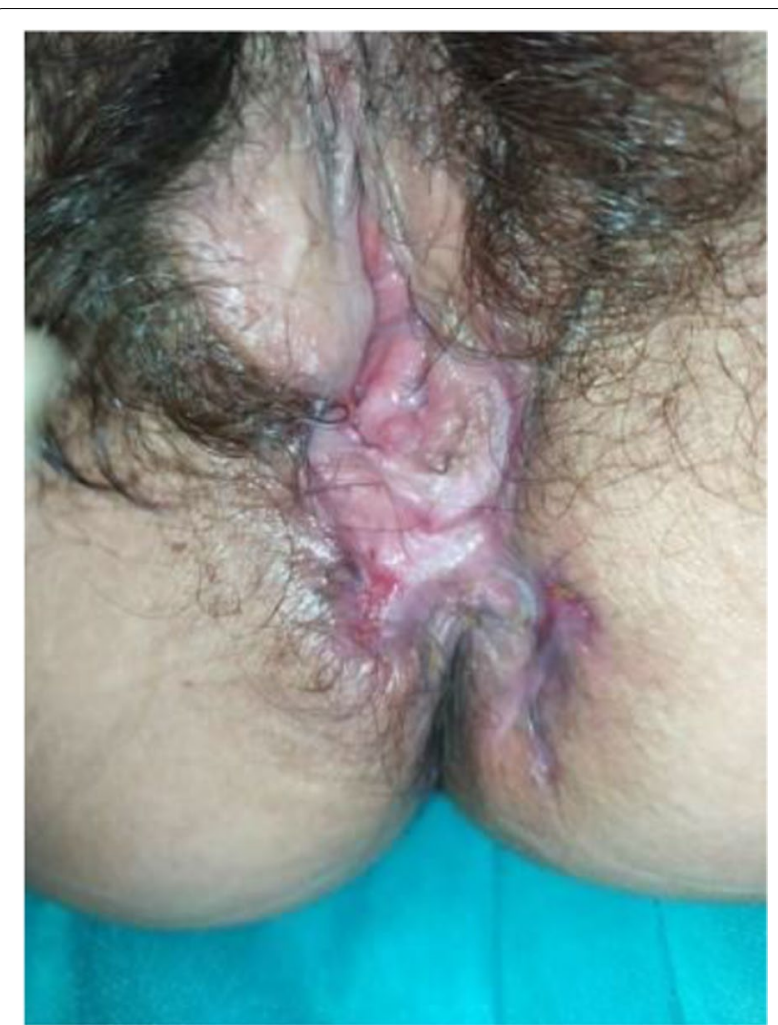

Fig. 1 The genital ulceration of the vulva with an extension to the perianal region before treatment

of ulcer showed neutrophilc infiltration without fibrin deposition or necrotic changes in vessel wall. Based on the clinical and histological finding, our case was diagnosed as genital ulcerative PG, which occurred during the exacerbation of BD and treated with colchicine at 1 mg per day, combined with corticosteroids at a rate of $0.5 \mathrm{mg} / \mathrm{kg}$ per day of prednisolone. Genital ulcer markedly healed, and oral aphtosis rapidely improved after one week of treatment with a normalization of CRP (Fig. 5). Because of the severe ocular involvement of the posterior segment, treatment with azathioprine at a rate of $100 \mathrm{mg} /$ day combined with Infliximab at a rate of 5 $\mathrm{mg} / \mathrm{kg}$ was initiated. The outcome was favorable with clinical and biological remission maintained with a follow-up of 6 months.

\section{Discussion}

The observation that we are reporting is unprecedented because of PG, which is a rare idiopathic inflammatory disease moreover localized on the genital sphere, and its equally rare association with Behçet's disease. PG was revealing a $B D$ in our patient.

In its typical form, the PG is characterized by one or many sterile painful ulcers, with a purulent base, raised

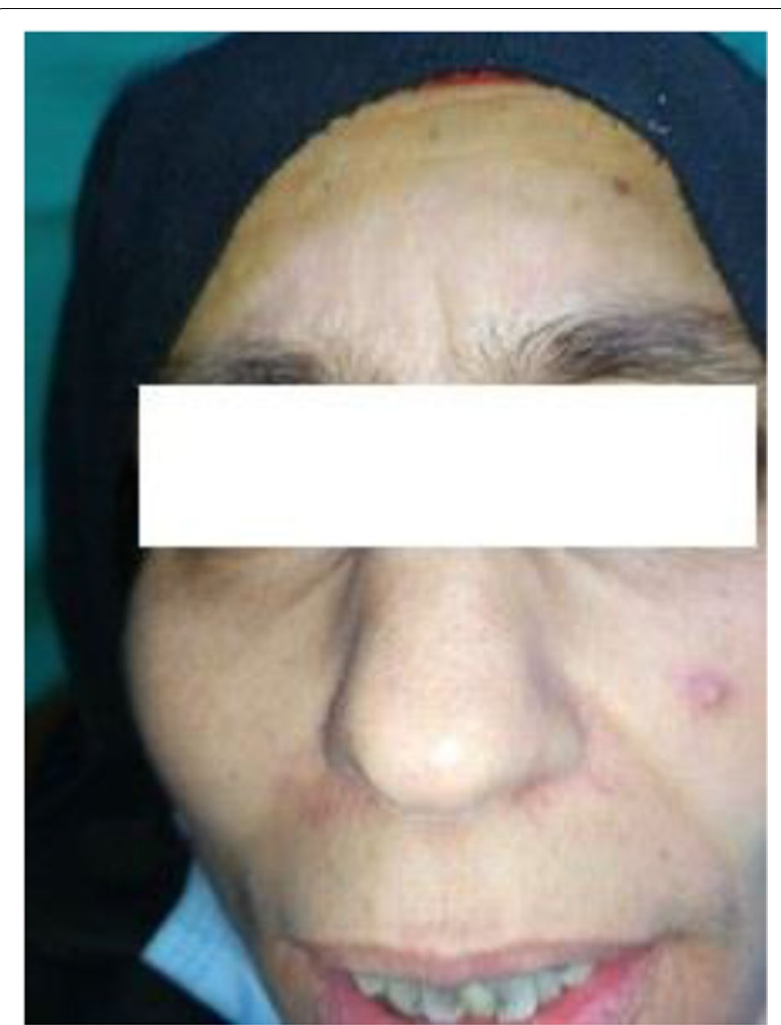

Fig. 2 A pseudo folliculitis on the left cheek

and undermined purplish edges; it has a rapid extension and a chronic evolution. There are several clinical forms, but the appearance is often suggestive of a subacute or a chronic infection, paradoxically, there is no lymphangitis or adenopathy. The pain is variable, it can sometimes be very intense [4]. The initial search for microorganisms is always negative but secondary infection occurs frequently [4]. The lower limbs constitute the preferred site of the PG. Other locations can be observed in particular on the buttocks, the cephalic extremity, and more rarely the anal and genital region [1]. The latter can be a source of diagnostic and therapeutic delay, which was the case with the patient.

PG is associated with systemic disease in approximately 50 to $78 \%$ of patients [5]. Inflammatory bowel disease, arthritis, and hematologic disease are the most common pathologic associations [5].

The association between PG and BD is rare. Since, the first case of Munro and Cox in 1988 [6], only 16 cases of PG associated with BD have been reported in the literature [6-21]. In the majority of these observations, PG is diagnosed after the associated disease [15]. Nevertheless, it can precede the onset, or be the telltale sign of an underlying disease [5]. 


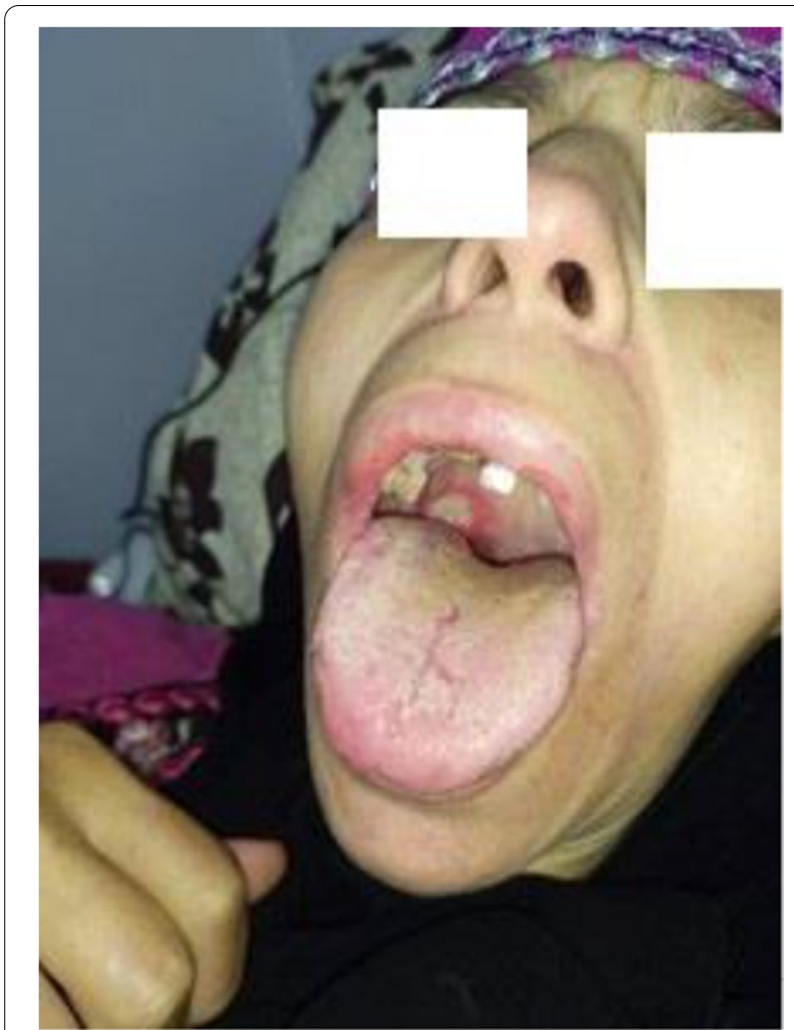

Fig. 3 Oral aphtosis on the palete

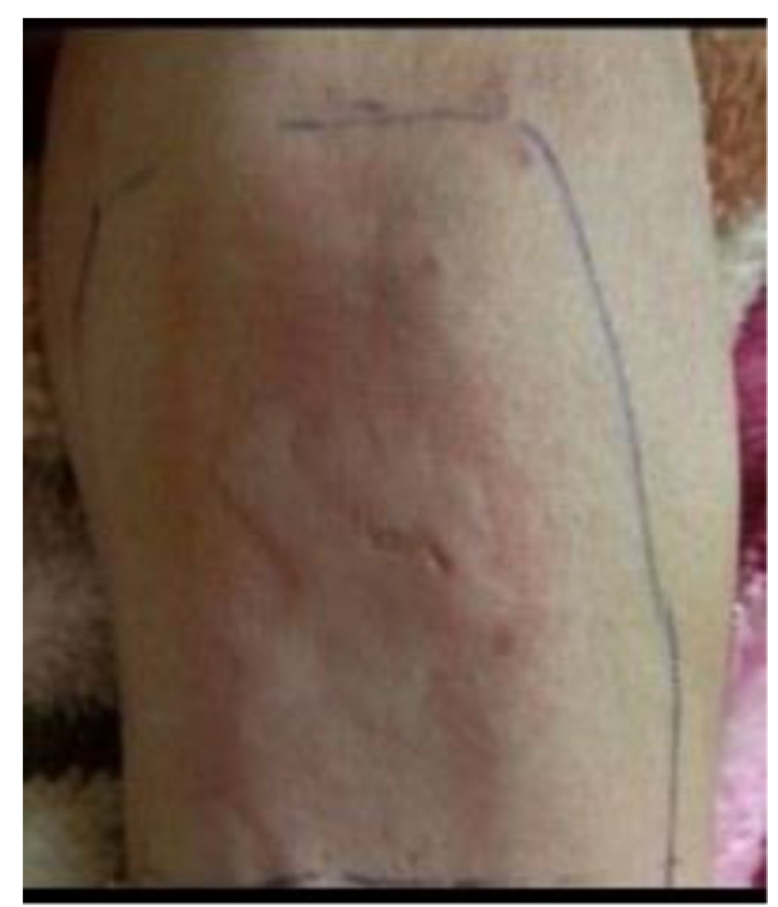

Fig. 4 The strongly positive pathergy test
Table 1 The international criteria for Behçet's disease scoring system. Point Score System : Scoring $\geq 4$ indicates Behcet's diagnosis) [3]

\begin{tabular}{ll}
\hline Sign/symptom & Points \\
\hline Ocular lesions & 2 \\
Genital aphthosis & 2 \\
Oral aphthosis & 2 \\
Skin lesions & 1 \\
Neurological manifestations & 1 \\
Vascular manifestations & 1 \\
Positive pathergy test & 1 \\
\hline
\end{tabular}

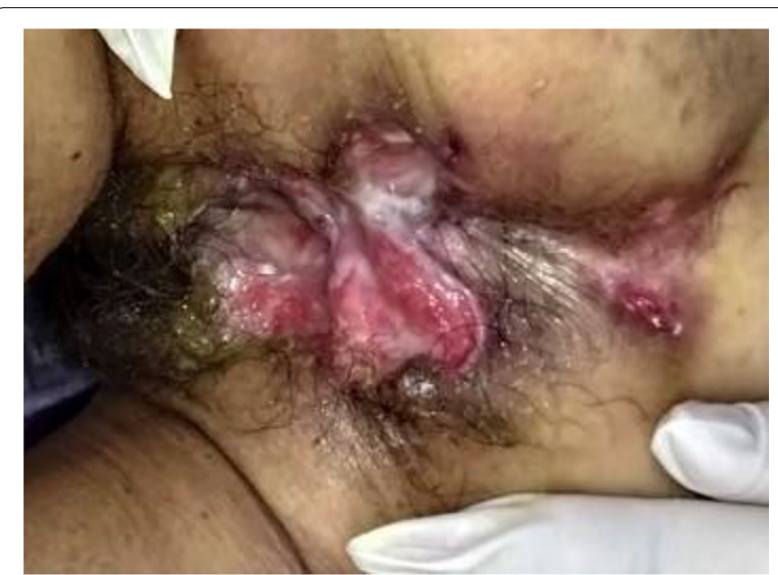

Fig. 5 Improvement of the genital ulceration after a week of treatement by corticosteroïdes

In the present case, genital ulcerative PG developed concurrently with an exacerbation of BD. We report the third case of PG in the literature, showing mucosal ulcers associated with BD $[8,17]$.

Behçet's disease was described by Hulusi Behçet as a multisystem inflammatory disease [22]. Currently, it is classified in the category of vasculitis affecting vessels of variable diameter [2]

Although oral ulceration, genital ulceration, and eye disease are the classic triad of manifestations, the cardiovascular, gastrointestinal, musculoskeletal, and central nervous systems can also be affected [3].

In $\mathrm{BD}$, multiple cutaneous lesions such as erythema nodosum, folliculitis, and pustules are seen, but PG is very rare [15].

The patient was diagnosed as Behcet's disease according to the criteria of the "International Study Group for Behçet's disease" [3]. She presented one major criteria (recurrent oral aphthosis) and three minor criteria (very suggestive ocular involvement, a presence of cutaneous pathergy and pseudo folliculitis lesions). The diagnosis of genital PG was based on the presence of two major 
criteria (appearance of the ulceration, exclusion of other causes of infectious or vascular skin ulcerations) and four minor criteria [23, 24] (positive pathergy reaction, rapid therapeutic response to systemic corticosteroid therapy, presence of a predominantly neutrophilic inflammatory infiltrate on histology, and association with BD) (Table 2).

It is sometimes difficult to distinguish between clinical manifestations of BD and PG in the same patient. Both $\mathrm{BD}$ and PG belong to the category of the neutrophilic dermatoses in which primary pustular lesions occur and neither of them has a characteristic histological appearance [15].

Indeed, at the onset stage and during the pustulo-ulcerative form of PG, the resemblance to skin lesions of $\mathrm{BD}$ is possible, and the pathergy test can be positive in both conditions [1].

The term pathergie refers to the occurrence of skin lesions following minimal trauma, and is used to assess excessive irritability of the skin in inflammatory diseases [23].

Its positivity in these two pathologies reflects the presence of an exaggerated inflammatory reaction. Histologically, this aberrant reaction leads to a release of cytokines responsible for a perivascular infiltrate and the accumulation of neutrophils at the site of the reaction [25].

Likewise, ulcerative lesions of the mouth and genital area can be seen in both diseases [19]. On the other hand, the frequency of mucosal ulcers and their clinical appearance are completely different [19].

Orogenital ulcers are commonly seen in BD, whereas rarely seen in PG. In BD, genital ulcers are similar in appearance to oral ulcers and can sometimes cause scar formation, and tend to be small and round, with wellcircumscribed margins, an erythematous halo and a yellow-grey floor. In PG, ulcers of the genital mucosa appear similar to those of skin, which are comprised of a burrowing ulcers with irregular margins and a ragged purple overhanging edges. Rapid local destruction may occur in PG [19]. Thus, the clinical features of mucosal ulcers are different to some degree between the two diseases.
However, there is a histopathological distinction between PG and BD. The two pathologies are characterized by a neutrophilic vascular reaction [15].

In PG, vascular involvement ranges from none to fibroid necrosis, and in most cases a neutrophilic infiltrate is present with limited vascular damage [19]. Conversely, in BD, mononuclear cell vasculitis with variable fibrin deposition or leukocytoclastic vasculitis is usually found [19].

$\mathrm{BD}$ has been associated with other neutrophilic dermatoses such as Sweet's syndrome and neutrophilic eccrine hidradenitis [1]. This suggests the presence of a clinical and histological overlap between $\mathrm{BD}$ and neutrophilic dermatoses. These overlaps could constitute the clinical expression of two autoinflammatory diseases in whose genetic component remains to be determined [1]. However, the true etiopathogenic link between these two conditions is not yet fully understood.

The activity of PG is chronic, and closely related to the activity of associated diseases [15].

Treatment of PG is usually based on systemic corticosteroid therapy alone or in combination with thalidomide or Dapsone [1]. In cases of corticosteroid-resistant PGs, the use of other immunosuppressants, such as tacrolimus, cyclosporin and mycophenolate mofetil, and azathioprine, may be effective. Currently, anti-TNFa are a promising treatment for refractory PG [1]. These therapeutic agents are used to treat both PG and BD [4].

The prognosis of PG depends on the associated disease and the severity of the clinical form of PG [1].

In this patient, the evolution of PG and oral aphthosis were favorable from the start of corticosteroid treatment. The use of immunosuppressive therapy combined with TNF inhibitors was justified by the presence of severe ocular involvement of the posterior segment.

\section{Conclusion}

The observation that we report is illustrated by both a rare localization of a PG (genital sphere), and its exceptional association with Behçet's disease. The coexistence of these two conditions could be a source of diagnostic

Table 2 PG diagnostic criteria [23, 24]

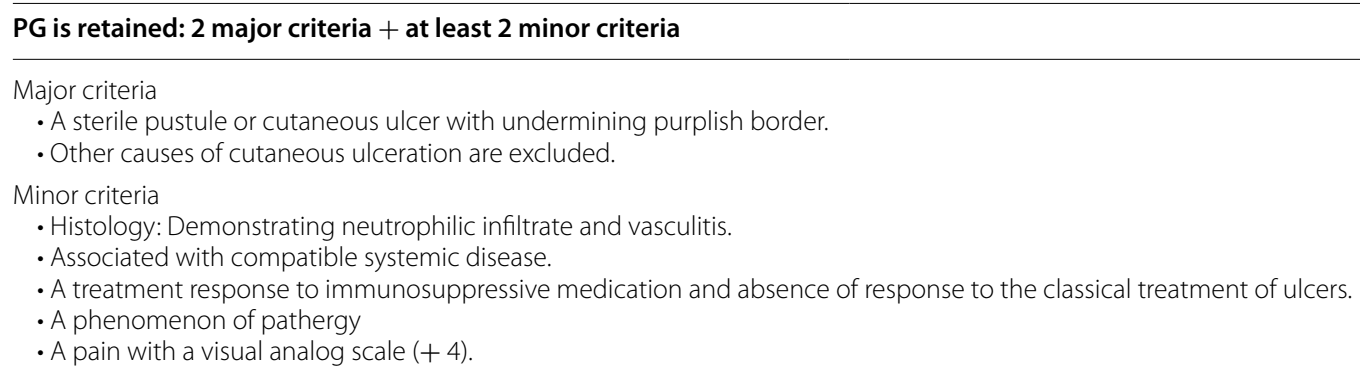


difficulties. The treatment and prognosis of PG are conditioned by the severity of the visceral involvement of $\mathrm{BD}$.

\section{Abbreviations}

PG: Pyoderma gangrenosum; BD: Behçets disease; CRP: Reactive protein.

\section{Acknowledgements}

Not applicable

\section{Authors' contributions}

S.T contributed to the writing of the article, its correction and the management of the patient. N.T, N.T and A.C contributed to the care of the patient in the department. All authors read and approved the final manuscript.

\section{Funding}

The authors have not received funding in any form.

\section{Availability of data and materials}

Not applicable (all the data of the reported case are available from the corresponding author).

\section{Declarations}

Ethics approval and consent to participate

Not applicable

\section{Consent for publication}

We obtained verbal consent from the patient. Our patient is illiterate.

\section{Competing interests}

The authors declare that they have no competing interests.

Received: 3 October 2021 Accepted: 15 November 2021

Published online: 11 December 2021

\section{References}

1. Hali F, Khadir K, Chiheb S, Bouayad K, Mikou N, Benchikhi H (2011) Pyoderma gangrenosum and Behcet's disease: a study of two pediatric cases. Arch Pediatr. 18(12):1320-1323

2. Jennette JC, Falk RJ, Bacon PA, Basu N, Cid MC, Ferrario F et al (2013) 2012 revised International Chapel Hill Consensus Conference Nomenclature of Vasculitides. Arthritis Rheumatism. 65(1):1-11

3. The International Criteria for Behçet's Disease (ICBD) (2014) The International Criteria for Behçet's Disease (ICBD): a collaborative study of 27 countries on the sensitivity and specificity of the new criteria. J Euro Acad Dermatol Venereol 28(3):338-347

4. Chariatte N, Lysitsa S, Lombardi T, Samson J, editors. (2011) Pyoderma gangrenosum (2ème partie) : manifestations buccales et présentation " un cas.

5. Ahronowitz I, Harp J, Shinkai K (2012) Etiology and management of pyoderma gangrenosum: a comprehensive review. Am J Clin Dermatol. 13(3):191-211

6. Munro CS, Cox NH. Pyoderma gangrenosum associated with Behçet's syndrome--response to thalidomide. Clin Exp Dermatol. 1988;13(6):408-410

7. Brunsting LA, Goeckerman WH, O'Leary PA. (1930) Pyoderma (echthyma) gangrenosum: clinical and experimental observations in five cases occurring in adults. Arch Dermatol Syphilol:22(4):655-680.

8. Lilford RJ, Tindall VR, Batchelor AG (1989) Post-surgical pyoderma gangrenosum of the vaginal vault associated with ulcerative colitis and Behcet's disease; a case report. Eur J Obstet Gynecol Reprod Biol. 31(1):93-94

9. Rustin MH, Gilkes JJ, Robinson TW (1990) Pyoderma gangrenosum associated with Behcet's disease: treatment with thalidomide. J Am Acad Dermatol. 23(5 Pt 1):941-944
10. Armas JB, Davies J, Davis M, Lovell C, McHugh N (1992) Atypical Behcet's disease with peripheral erosive arthropathy and pyoderma gangrenosum. Clin Exp Rheumatol. 10(2):177-180

11. Chams-Davatchi C, Shizarpour M, Davatchi F, Shahram F, Chams H, Nadji A et al (2003) Extensive pyoderma gangrenosum-like lesion in two cases of Behcet's disease, responding only to cyclosporin. Advances in experimental medicine and biology. 528:337-338

12. Joshi A, Mamta. (2004) Behcet's syndrome with pyoderma-gangrenosumlike lesions treated successfully with dapsone monotherapy. J Dermatol. 31(10):806-810

13. Singh G, Sethi A, Okade R, Harish MR (2005) Bullous pyoderma gangrenosum: a presentation of childhood Behcet's disease. Int J Dermatol. 44(3):257-258

14. Akay N, Boyvat A, Heper AO, Soykan I, Arica IE, Bektas M et al (2006) Behcet's disease-like presentation of bullous pyoderma gangrenosum associated with Crohn's disease. Clin Exp Dermatol. 31(3):384-386

15. Nakamura T, Yagi H, Kurachi K, Suzuki S, Konno H (2006) Intestinal Behcet's disease with pyoderma gangrenosum: a case report. World J Gastroenterol. 12(6):979-981

16. Kim JW, Park JH, Lee D, Hwang SW, Park SW (2007) Vegetative pyoderma gangrenosum in Behcet's disease. Acta Derm Venereol. 87(4):365-367

17. Tsuboi H (2008) Case of pyoderma gangrenosum showing oral and genital ulcers, misdiagnosed as Behcet's disease at first medical examination. J Dermatol. 35(5):289-292

18. Kashiwado Y, Uchino A, Ota T, Nagano S (2018) Intestinal Behcet's disease with pyoderma gangrenosum successfully treated with the combination therapy of adalimumab and glucocorticoids. Mod Rheumatol. 28(5):901-905

19. Ozuguz P, Kacar SD, Manav V, Karaca S, Aktepe F, Ulu S (2015) Genital ulcerative pyoderma gangrenosum in Behcet's disease: a case report and review of the literature. Indian J Dermatol. 60(1):105

20. Yusuke Kashiwado, Ayumi Uchino, Toshiyuki Ota, Shuji Nagano. (2016) Intestinal Behçet's disease with pyoderma gangrenosum successfully treated with the combination therapy of adalimumab and glucocorticoids. Mod Rheumatol. Early Online: 1-5.

21. J.L. Patier de la Penã, M.Á. Moreno-Cobo,M. Sánchez-Conde y A.M. Echaniz Quintana Behçet's and refractory pyoderma gangrenosum with response to infliximab. Rev Clin Espanola. 215(1): 66-67

22. (1990) Groupe d'étude international sur la maladie de Behçet. Critères de diagnostic de la maladie de Behçet. Groupe d'étude international sur la maladie de Behçet. Lancette. 335:1078-1080

23. Wollina U, Tchernev G. Pyoderma gangrenosum: pathogenetic oriented treatment approaches. Wiener medizinische Wochenschrift (1946). 2014;164(13-14):263-273.

24. Modiano P (2015) Actualité sur la prise en charge du pyoderma gangrenosum en 2015. Annales de Dermatologie et de Vénéréologie. 142(8):502-505

25. Rahman S, Daveluy S (2021) Pathergy Test. StatPearls, Treasure Island

\section{Publisher's Note}

Springer Nature remains neutral with regard to jurisdictional claims in published maps and institutional affiliations. 